Zeneca, unrelated to this project., Grant/research support from: Gilead, unrelated to this project.

DOI: 10.1136/annrheumdis-2021-eular.1032

\section{OP0118 EFFECT OF WITHDRAWING ETANERCEPT OR METHOTREXATE ON PATIENT-REPORTED OUTCOMES IN RHEUMATOID ARTHRITIS PATIENTS IN REMISSION ON COMBINATION THERAPY: RESULTS FROM THE SEAM-RA TRIAL}

J. Curtis ${ }^{1}$, E. Karis ${ }^{2}$, V. Bykerk ${ }^{3}$, G. Kricorian ${ }^{4}$, P. Yen ${ }^{5}$, P. Emery ${ }^{6}$, P. Haraoui ${ }^{7}$, D. Collier ${ }^{4}$, B. Stolshek ${ }^{8} .{ }^{1}$ University of Alabama at Birmingham, Division of Clinical Immunology and Rheumatology, Birmingham, United States of America; ${ }^{2}$ Amgen Inc, Global Development, California, United States of America: ${ }^{3}$ Hospital for Special Surgery, Department of Rheumatology, New York, United States of America; ${ }^{4}$ Amgen Inc, Global Medical, California, United States of America: ${ }^{5}$ Amgen Inc, Global Biostatistical Science, California, United States of America; ${ }^{6}$ Leeds National Institute for Health Research, Biomedical Research Center at Leeds Teaching Hospitals Trust, LIRMM Leeds University, Division of Rheumatic and Musculoskeletal Disease, Leeds, United Kingdom ${ }^{7}$ Rheumatology Institute of Montreal, Department of Medicine, Montreal, Canada; ${ }^{8}$ Amgen Inc, Global Health Economics, California, United States of America

Background: Limited studies have assessed the effect of withdrawal of either methotrexate (MTX) or etanercept (ETN) on patient-reported outcomes (PROs) in rheumatoid arthritis (RA).

Objectives: To evaluate the baseline and change in PROs following withdrawal of MTX or ETN in RA patients with sustained remission receiving combination ETN+MTX.

Methods: Adult patients with RA on ETN+MTX and in remission (SDAl $\leq 3.3$ ) for $\geq 12$ months (including a 24-week, open-label, run-in period) were randomized to a 48-week double-blind period to receive ETN $50 \mathrm{mg}$ weekly $(\mathrm{N}=101)$, oral MTX $10-25 \mathrm{mg}$ weekly $(\mathrm{N}=101)$ or continue $\mathrm{ETN}+\mathrm{MTX}(\mathrm{N}=51)$. The primary endpoint was maintenance of SDAl remission without disease worsening (DW) at week 48 between ETN and MTX groups. Patients who experienced SDAI >11 at any time after randomization, or SDAI $>3.3$ and $\leq 11$ during 2 consecutive or on 3 non-consecutive visits were considered to have DW and resumed ETN+MTX. PROs assessed were patient global assessment of disease activity (PtGA, 0-100 mm), patient joint pain (PtJP, 0-100 mm), Health Assessment Questionnaire-Disability Index (HAQ-DI), and the 36-item short-form health survey (SF-36) component and domain scores. A 2-sample t-test was used to compare the treatment differences between groups. A subgroup analysis for patients with DW was also performed (DW analysis set) and compared PROs between ETN vs MTX arms (ETN+MTX not shown given the small sample size).

Results: Of the 253 patients randomized, 121 (47.8\%) experienced DW and were included in the DW analysis set. Baseline demographics were generally balanced between the 3 treatment groups. Most patients were women (76.3\%), White $(87.0 \%)$, and with a mean age of 55.6 years. The mean (SD) MTX dose was $16.3(4.69) \mathrm{mg}$ and the mean (SD) duration of RA was 10.3 (7.8) years. At week 48, a significantly greater proportion of patients on ETN vs MTX monotherapy maintained SDAI remission $(49.5 \%$ vs $28.7 \%$; $P=0.004)$ after therapy withdrawal. In the overall population, PtGA and PtJP scores were very low at baseline (PtGA-MTX: 4.4, ETN: 4.5, ETN+MTX: 3.5; PtJP-MTX: 4.9, ETN: 5.5, ETN+MTX: 3.5 ) and showed some worsening over the study period in all treatment groups, with a mean change at week 48 ranging from 5.0 to 10.0 units for PtGA and 3.7 to 8.1 units for PtJP. Patients on ETN had less worsening, with a nominally significant treatment difference observed between ETN and MTX monotherapy groups for PtGA at almost all timepoints, and for PtJP at weeks 12 and 36 (Figure). Mean HAQ-DI (MTX: 0.32; ETN: 0.26; ETN+MTX: 0.28) and SF-36 scores (physical component [PCS]-MTX: 52.1, ETN: 52.7, ETN+MTX: 52.3; mental component [MCS]-MTX: 55.5, ETN: 55.8, ETN+MTX: 57.1) at baseline show that patients had low disability and excellent health-related quality of life compared with normative values for the general non-RA population. HAQ-DI scores were well maintained at weeks 24 and 48 (change from baseline at week 48-MTX: 0.14; ETN: 0.15; ETN+MTX: 0.21). The SF-36 PCS, MCS, and domain scores decreased minimally from baseline with treatment differences that were not nominally significant between groups. Among patients with DW during the study, those on ETN showed less PtGA and PtJP worsening from baseline than those on MTX at weeks 12, 36, and 48 (Figure). Other PROs (HAQ-DI [change from baseline at week 24-ETN: 0.34; MTX: 0.21; at week 48-ETN: 0.15; MTX: $0.15]$, SF-36 PCS, MCS, and domain scores) showed a similar degree of worsening in both the MTX and ETN arms.

Conclusion: In patients with sustained SDAI remission on ETN+MTX, mental and physical health as measured by SF-36 was comparable with that of the non-RA population. Withdrawal of ETN (MTX monotherapy) resulted in a greater worsening of PtGA and PtJP than withdrawal of MTX (ETN monotherapy), and patients on ETN monotherapy restored these scores close to baseline towards the end of the treatment period. These findings demonstrate that ETN monotherapy has a greater effect on maintaining overall patient assessment of disease and joint pain compared with MTX monotherapy.
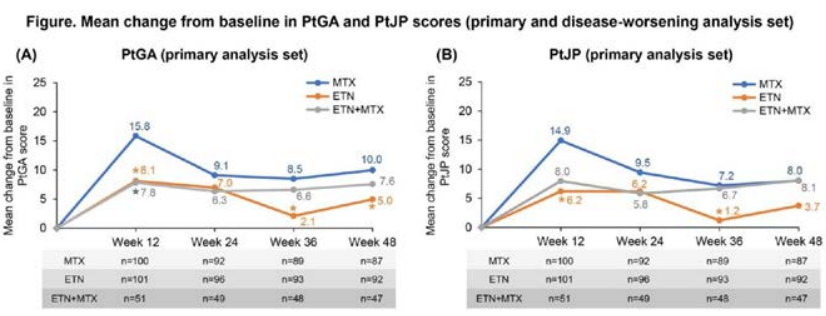

(C) PtGA (disease-worsening analysis set)

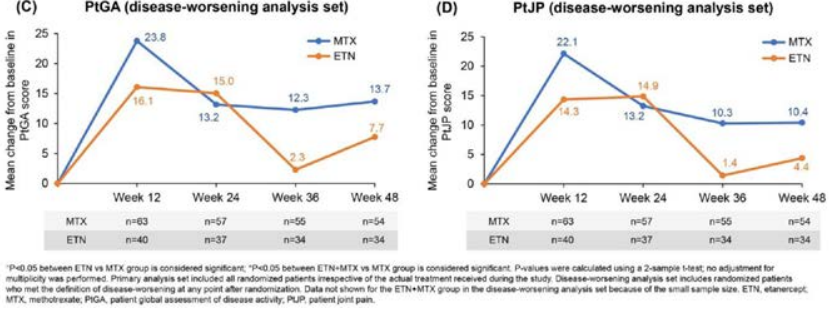

Disclosure of Interests: Jeffrey Curtis Speakers bureau: AbbVie, BMS, Gilead, Lilly, Novartis, Sanofi, Scipher, Amgen, Corrona, Janssen, Myriad, and Pfizer, Consultant of: AbbVie, BMS, Gilead, Lilly, Novartis, Sanofi, Scipher, Amgen, Corrona, Janssen, Myriad, and Pfizer, Grant/research support from: AbbVie, BMS, Gilead, Lilly, Novartis, Sanofi, Scipher, Amgen, Corrona, Janssen, Myriad, and Pfizer, Elaine Karis Shareholder of: Amgen Inc., Employee of: Amgen Inc., Vivian Bykerk Speakers bureau: Amgen, BMS, Gilead, Pfizer, Sanofi-Genzyme/Regeneron, Scipher Medicine, and UCB., Consultant of: Amgen, BMS, Gilead, Pfizer, Sanofi-Genzyme/Regeneron, Scipher Medicine, and UCB. Grant/research support from: Amgen and Novartis, Greg Kricorian Shareholde of: Amgen Inc., Employee of: Amgen Inc., Priscilla Yen Shareholder of: Amgen Inc., Employee of: Amgen Inc., Paul Emery Speakers bureau: AbbVie, BMS Celltrion, Gilead, Lilly, MSD, Novartis, Pfizer, Roche, Samsung, Sandoz, and UCB., Consultant of: AbbVie, BMS, Celltrion, Gilead, Lilly, MSD, Novartis, Pfizer Roche, Samsung, Sandoz, and UCB., Paul Haraoui Speakers bureau: AbbVie, Celgene, Janssen, Pfizer, and UCB., Consultant of: AbbVie, Amgen, BMS Celgene, Eli Lilly, Janssen, Merck, Pfizer, Roche, Sandoz, Sanofi-Genzyme, and UCB., Grant/research support from: Roche, AbbVie, Amgen, Merck, and Pfizer, David Collier Shareholder of: Amgen Inc., Employee of: Amgen Inc., Brad Stolshek Shareholder of: Amgen Inc., Employee of: Amgen Inc. DOI: 10.1136/annrheumdis-2021-eular. 1863

\section{OP0119 \\ SHOULD WE USE BIODMARDS IN FIRST INTENTION IN EARLY RHEUMATOID ARTHRITIS?: RESULTS AT 5 YEARS FROM THE ERA LOUVAIN BRUSSELS COHORT}

E. Sapart ${ }^{1}$, T. Sokolova ${ }^{1}$, S. De Montjoye ${ }^{1}$, S. Dierckx ${ }^{2}$, A. Nzeusseu Toukap ${ }^{1}$, A. Avramovska ${ }^{1}$, L. Meric de Bellefon ${ }^{1}$, P. Durez ${ }^{1} .{ }^{1}$ Cliniques Universitaires Saint-Luc - Université catholique de Louvain (UCL) - Institut de Recherche Expérimentale et Clinique (IREC), Rheumatology, Brussels, Belgium; ${ }^{2} \mathrm{CHU}$ Mont-Godinne, Rheumatology, Mont-Godinne, Belgium

Background: Early effective treatment has led to major improvements in patients with rheumatoid arthritis (ERA). Low disease activity and remission are achieved earlier and in higher frequency when the initial treatment in rheumatoid arthritis includes a combination of methotrexate (MTX) with a bDMARD compared to MTX alone.

Objectives: The aim of this study is to retrospectively analyse and compare the benefit of a treatment with methotrexate (MTX) alone or combined with a bDMARD as an induction therapy during 5 years of follow-up in early rheumatoid arthritis (ERA) patients.

Methods: We included ERA patients from the UCLouvain Brussels cohort who met the ACR/EULAR 2010 classification criteria and were naïve to DMARDs. Treatments were initiated based on the decision of a senior rheumatologist bDMARDs induction therapy was usually limited to 6 or 12 months. We collected patient characteristics at baseline and clinical response was analysed at 6 months, 1 year, 3 years and 5 years.

Results: Data from 470 eligible ERA patients were collected. The average age of the population is 48.9 years; $70.5 \%$ of the patients are women; $27.3 \%$ are smokers and $68.8 \%$ are positive for anti-citrullinated protein antibody (ACPA). 